\title{
High prevalence of cardiovascular risk factors in Durban South African Indians: The Phoenix Lifestyle Project
}

\author{
D R Prakaschandra, ${ }^{1} \mathrm{PhD}$, MMedSci; T M Esterhuizen, ${ }^{2} \mathrm{MSc}$; A A Motala, ${ }^{3} \mathrm{MB}$ ChB, MD, FRCP; P Gathiram, ${ }^{4} \mathrm{PhD}, \mathrm{MSc}$; \\ D P Naidoo, ${ }^{5} \mathrm{MB} \mathrm{ChB}, \mathrm{MD}, \mathrm{FRCP}$ \\ ${ }^{1}$ Department of Biomedical and Clinical Technology, Faculty of Health Sciences, Durban University of Technology, South Africa \\ ${ }^{2}$ Centre for Evidence-Based Health Care, Faculty of Medicine and Health Sciences, Stellenbosch University, Tygerberg, Cape Town, South Africa \\ ${ }^{3}$ Department of Diabetes and Endocrinology, School of Clinical Medicine, College of Health Sciences, Nelson R Mandela School of Medicine, \\ University of KwaZulu-Natal, Durban, South Africa \\ ${ }^{4}$ Department of Physiology, School of Clinical Medicine, College of Health Sciences, Nelson R Mandela School of Medicine, University of \\ KwaZulu-Natal, Durban, South Africa \\ ${ }^{5}$ Department of Cardiology, School of Clinical Medicine, College of Health Sciences, Nelson R Mandela School of Medicine, University of \\ KwaZulu-Natal, Durban, South Africa
}

Corresponding author: D P Naidoo (naidood@ukzn.ac.za)

\begin{abstract}
Background. Previous studies show a high prevalence of cardiovascular (CV) risk factors in South African (SA) Asian Indians, with the emergence of premature coronary artery disease in young Indian subjects.

Objective. To determine the prevalence of $\mathrm{CV}$ risk factors in this population.

Methods. This was a cross-sectional study of randomly selected adults aged 15 - 64 years from the suburb of Phoenix in Durban, KwaZulu-Natal Province, SA. All participants had demographic, anthropometric and biochemical measurements using the modified World Health Organization (WHO) STEPwise survey methods. Hypertension, obesity, lipid abnormalities and diabetes mellitus (DM) were diagnosed using WHO criteria. Age-standardised frequencies for glycaemic indices were calculated according to the WHO standard world population distribution.

Results. Of the 1428 subjects who responded (response rate 72.1\%), complete data for analysis were available on 1378 (1 001 women). The mean age was 45.5 (standard deviation 13) years. There were high prevalences of hypertension (47.5\%), DM (20.1\%), total body obesity (raised body mass index) (32.4\%) and increased waist circumference (73.1\%). The 'thin-fat' Asian phenotype (isolated abdominal obesity) was found in only $4.8 \%$ of participants. High prevalences of total body obesity $(32.1 \%)$, increased waist circumference (31.3\%) and insulin resistance $(28.2 \%)$ were documented in the youngest age group. Over half of the males and $14.6 \%$ of females were current smokers. Diabetic dyslipidaemia was found in 61 subjects (4.4\%). In multivariate analysis, age, triglycerides and waist circumference measurement were significant independent risk factors associated with DM and, together with fasting glucose, also predicted hypertension.

Conclusion. Compared with Asian Indian subjects with similar environmental exposure in previous studies, the magnitude of change in risk factor prevalence over the past two decades has been of epidemic proportions.
\end{abstract}

S Afr Med J 2016;106(3):284-289. DOI:10.7196/SAMJ.2016.v106i3.9837

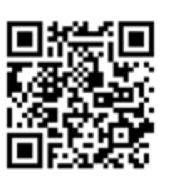

The latest data from the International Diabetes Federation ${ }^{[1]}$ show that the prevalence of adults with type 2 diabetes mellitus (DM) is highest in low- and middle-income countries, with sub-Saharan Africa and the Indian subcontinent being highlighted as two of the regions where this rise will be the greatest. Earlier epidemiological studies ${ }^{[2,3]}$ show that South African (SA) Indians have a high prevalence ${ }^{[2]}$ and incidence ${ }^{[3]}$ of DM. In the past two decades there have been major sociopolitical changes in these communities, and the lack of current data on the changes in cardiovascular $(\mathrm{CV})$ risk profile and the emergence of premature coronary artery disease ${ }^{[4]}$ was the rationale for a survey of the $\mathrm{CV}$ risk factor profile of SA Indians.

\section{Objective}

To determine the CV risk factor prevalence in a community-based sample of SA Indians.

\section{Methods \\ Sampling}

The Phoenix Lifestyle Project was a cross-sectional study conducted over a 2-year period (January 2007 - December 2008) on SA Indians aged 15 - 64 years residing in the Phoenix area (eThekwini Municipality,
Durban, SA). This community forms a heterogeneous population of Dravidian and Aryan ancestry, and are mainly descendants of Indians who arrived in SA from 1860 as indentured labourers on the sugar plantations in Natal. ${ }^{[5]}$

Two-stage cluster sampling was used to select the households. First, a proportional representation of the number of households was selected from each area. Thereafter, the households to be studied in each area were randomly selected. One individual from each household was selected using the Kish method ${ }^{[6]}$ Fieldworkers were trained to follow a prescribed guideline using the Kish method of selection so that participants were stratified for age (15 - 24, 25 - 34, 35 - 44, 45 - 54 and 55 - 64 years) and gender (male and female), with 198 males and 198 females in each stratum.

\section{Ethical considerations}

The study was approved by the University of KwaZulu-Natal Bioethics Committee (ethics reference: E336/05) and conformed to the principles in the Declaration of Helsinki. All subjects gave written informed consent for the collection of the data and were informed of the results of the examinations undertaken. Subjects in whom risk factors were identified were referred to a health facility for further evaluation and management. 


\section{Data collection}

The World Health Organization (WHO) modified STEPS questionnaire for non-communicable disease risk factors (version 1.4 questionnaire) ${ }^{[7]}$ was used to record the demographic (including age, sex, education and income level) information and behavioural measurements at the participants' homes. Other information included type of diet, physical activity, smoking habits, alcohol consumption and a personal and family (first-degree relatives) history of DM, hypertension and CV risk factors.

The clinical examination and investigations were performed at the Lifestyle Centre, Inkosi Albert Luthuli Central Hospital, Durban. Anthropometric measurements performed included height, weight and waist circumference according to WHO criteria. ${ }^{[7]}$ Venous blood samples were taken after an overnight fast for measurement of plasma glucose, serum insulin and serum lipid (total cholesterol, highdensity lipoprotein (HDL) cholesterol and total triglyceride) levels. Fasting plasma glucose was measured using the glucose oxidase method and plasma insulin was measured by immunoassay. A $75 \mathrm{~g}$ oral glucose tolerance test (OGTT) was performed on participants who did not self-report a positive history of DM, for fasting and 2-hour plasma glucose levels. Blood pressure (BP) was recorded on three occasions at 2-minute intervals and the average of the three readings was documented.

\section{Definition and classification of $\mathrm{CV}$ risk factors Anthropometry}

Based on the body mass index (BMI), participants were classified as of normal weight $\left(18.50-24.99 \mathrm{~kg} / \mathrm{m}^{2}\right)$, overweight $\left(\geq 25-29.99 \mathrm{~kg} / \mathrm{m}^{2}\right)$ or obese $\left(\geq 30 \mathrm{~kg} / \mathrm{m}^{2}\right)$. The revised cut-off point for Asian Indians ${ }^{[8]}$ was also applied, i.e. subjects with a BMI $<23 \mathrm{~kg} / \mathrm{m}^{2}$ were classified as normal, those with a BMI of $23.0-24.9 \mathrm{~kg} / \mathrm{m}^{2}$ as overweight and those with a BMI of $\geq 25.0 \mathrm{~kg} / \mathrm{m}^{2}$ as obese. The prevalence of the 'thin-fat Asian phenotype' was also determined in subjects with isolated abdominal obesity, i.e. Asian criteria for waist circumference (men $>90 \mathrm{~cm}$, women $>80 \mathrm{~cm})$ and a normal BMI $\left(<23 \mathrm{~kg} / \mathrm{m}^{2}\right)$.

\section{Hypertension}

Individuals who self-reported previously diagnosed hypertension, and/or those with blood pressure readings $\geq 140$ and $\geq 90 \mathrm{mmHg}$ (average of three readings) (Joint National Committee VII (JNC VII) criteria) and/or those on current antihypertensive therapy ${ }^{[9]}$ were defined as having systemic hypertension. Using JNC VII guidelines, individuals with a systolic blood pressure of $120-139 \mathrm{mmHg}$ or a diastolic blood pressure of $80-89 \mathrm{mmHg}^{[9]}$ were classed as having prehypertension.

\section{Biochemistry}

Lipid parameters were classified using criteria of the National Cholesterol Adult Panel III guidelines: ${ }^{[10]}$ serum total cholesterol $>5.17 \mathrm{mmol} / \mathrm{L}$, serum total triglyceride $>1.69 \mathrm{mmol} / \mathrm{L}$, HDL cholesterol $<1.04 \mathrm{mmol} / \mathrm{L}$ (men) and $<1.29 \mathrm{mmol} / \mathrm{L}$ (women), and low-density lipoprotein (LDL) cholesterol $>2.59 \mathrm{mmol} / \mathrm{L}$. LDL cholesterol was calculated using the Friedewald equation. Diabetic dyslipidaemia was diagnosed if raised levels of LDL cholesterol and triglycerides, as well as low levels of HDL cholesterol, were present.

The WHO criteria ${ }^{[11]}$ were used to classify glycaemic categories based on fasting plasma glucose (FPG): DM if the FPG was $\geq 7.0 \mathrm{mmol} / \mathrm{L}$, and impaired fasting glucose (IFG) if the FPG was $\geq 6.1$ and $\leq 6.9 \mathrm{mmol} / \mathrm{L}$. Impaired glucose tolerance was defined if the FPG was $<7.0 \mathrm{mmol} / \mathrm{L}$ and the 2-hour plasma glucose level during the OGTT was $\geq 7.8$ and $<11.1 \mathrm{mmol} / \mathrm{L}$. Subjects with IFG or IGT were classified as having prediabetes. Homeostatic model assessment for insulin resistance (HOMA-IR) values were calculated using the formula: fasting insulin $(\mu \mathrm{U} / \mathrm{m}) \times$ fasting glucose $(\mathrm{mmol} / \mathrm{L}) / 22.5$. Insulin resistance was defined as a HOMA-IR value of $>2.6 .^{[12]}$ The age-standardised frequencies for glycaemic indices were calculated according to the WHO standard world population distribution.

\section{Statistical analysis}

Statistical analysis was performed using the SPSS programme (version 22, SPSS, USA). The distributional properties for all variables were investigated, and variables with a skewed distribution were logtransformed or analysed using non-parametric testing. Means and standard deviations (SDs) and median values with interquartile ranges for non-Gaussian distributed variables were calculated for continuous variables. Categorical data were presented as percentages with corresponding $95 \%$ confidence intervals (CIs); the $\chi^{2}$ test was used for intergroup comparisons. For continuous variables, analysis of variance was used. Post-hoc analysis of variance comparisons for continuous variables was based on the Bonferroni correction.

Logistic regression (stepwise, backward) was used to identify risk factors associated with DM and hypertension. A $p$-value of $<0.05$ was regarded as being statistically significant.

\section{Results}

Of 1980 participants selected, 1428 individuals (402 males, 1026 females) participated in the study, giving a response rate of $72.1 \%$. Non-responders were mainly males (40.6\%, 402/990 male participants selected), with participation being higher in the older age groups. Complete data on all variables were available on 1378 participants, who formed the study group for further analysis shown in Table 1. The majority of respondents were women ( $n=1001,72.6 \%)$, underrepresented in the 15 - 34-year age groups and over-represented in the 35 - 64-year age groups (Table 1).

The mean age of the total sample was 45.5 (SD 13) years, with the highest proportion of subjects in the 45 - 54-year age group (30.7\%) (Table 2). The general pattern for blood pressure and biochemical variables among both males and females was an increase with advancing age. Participants in all age groups reported a positive family history for one or more of the following conditions: obesity, $\mathrm{DM}$, hypertension, myocardial infarction (MI), stroke and heart failure (congestive heart failure (CHF), with hypertension being the most frequently reported (71.2\%).

\section{$\mathrm{CV}$ and metabolic risk factors in the sample}

The most frequent abnormality was adiposity, both total (BMI) and central obesity (waist circumference) (Table 3). Almost onethird were classified as obese $(32.4 \%)$ or overweight $(32.0 \%)$, with the prevalence increasing with age $(p$-trend $<0.001)$. Using Asian cut-points, the prevalence of obesity doubled to $64.5 \%$, and was significantly higher in women (71.0\%) than in men $(44.5 \%)$

Table 1. Age and gender distribution of study subjects

\begin{tabular}{llll}
\hline Age group (years) & $\begin{array}{l}\text { Total, } \\
\boldsymbol{n}(\%)\end{array}$ & $\begin{array}{l}\text { Males, } \\
\boldsymbol{n}(\%)\end{array}$ & $\begin{array}{l}\text { Females, } \\
\boldsymbol{n}(\%)\end{array}$ \\
\hline $15-24$ & $131(9.5)$ & $54(41.2)$ & $77(58.8)$ \\
$25-34$ & $161(11.6)$ & $60(37.3)$ & $101(62.7)$ \\
$35-44$ & $295(21.4)$ & $68(23.1)$ & $227(76.9)$ \\
$45-54$ & $423(30.7)$ & $81(20.1)$ & $342(80.9)$ \\
$55-64$ & $368(26.7)$ & $114(30.9)$ & $254(69.0)$ \\
Total & 1378 & $377(27.3)$ & $1001(72.6)$
\end{tabular}




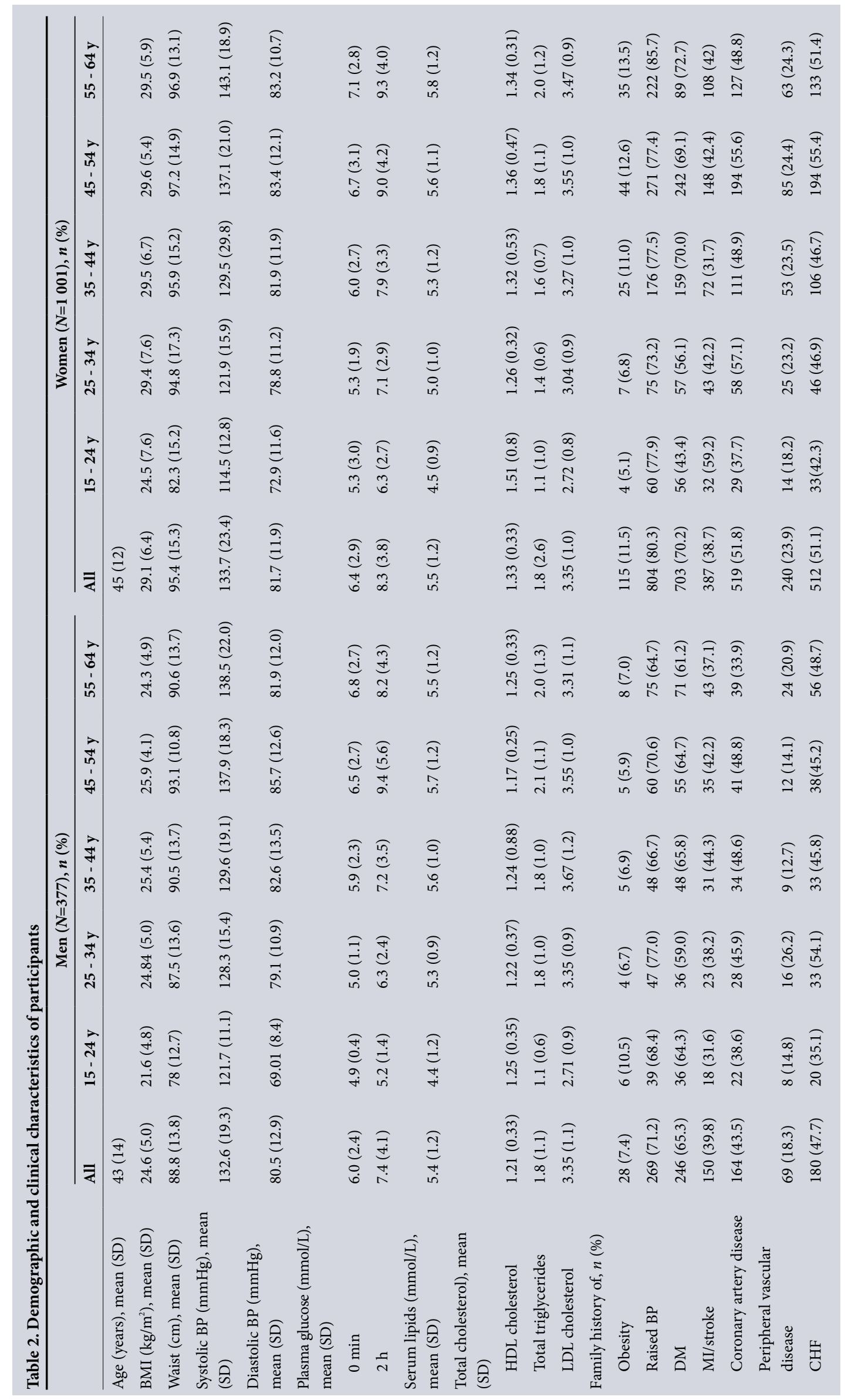




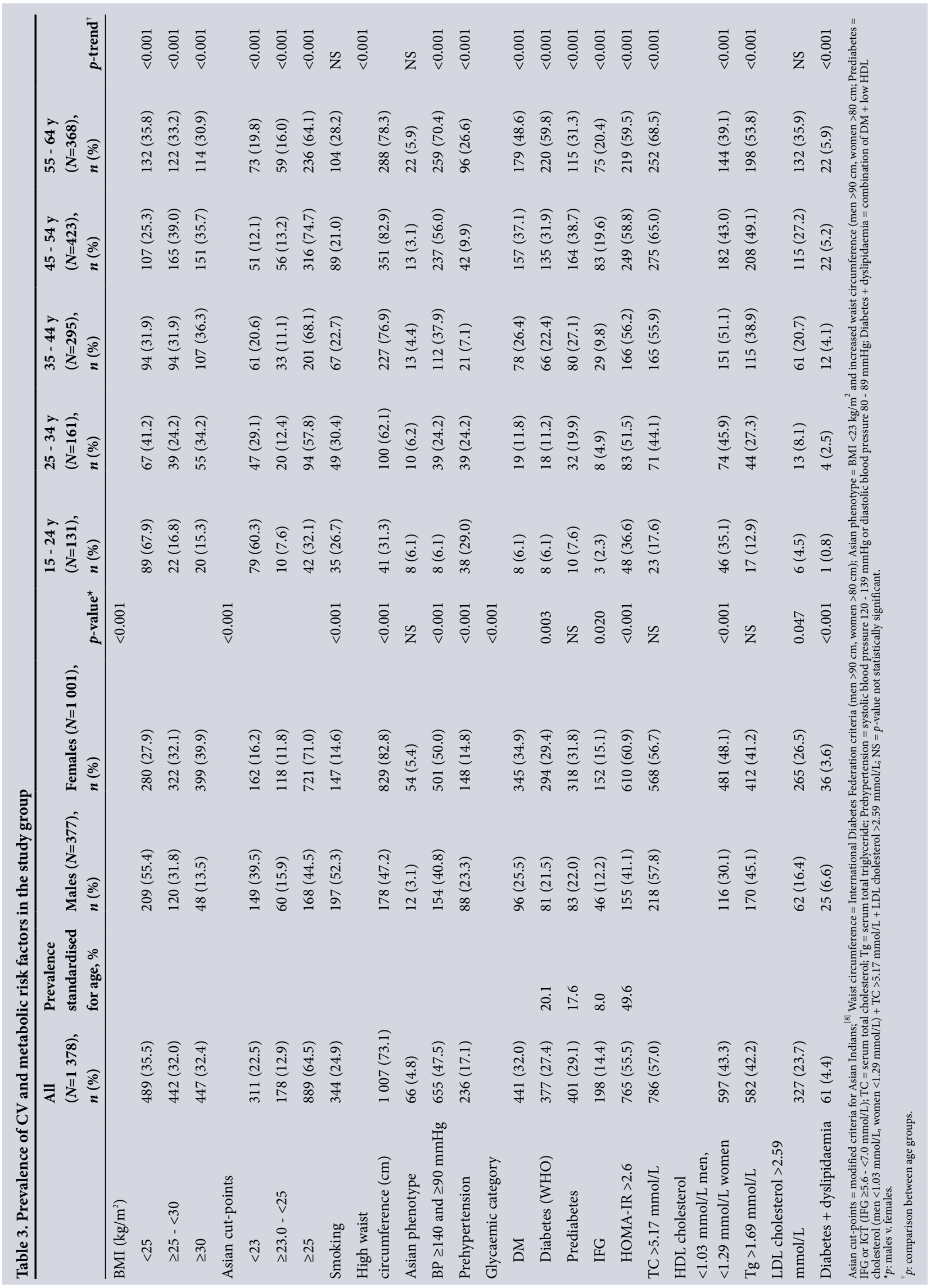


$(p<0.001)$; of interest, almost a third of the participants (32.1\%) in the 25 - 34-year age groups were also obese. Isolated abdominal obesity using the Asian cut-points for waist circumference and a normal BMI (the 'Asian phenotype') was recorded in only $4.8 \%$ of the sample.

Almost half (47.5\%) of the total group had hypertension, with the prevalence higher in women than in men $(p<0.001)$ and increasing with age $(p<0.001)$; the peak prevalence was in the oldest age groups. Prehypertension was found in $17.1 \%(n=236)$ of participants, and was more common in men than in women.

The crude prevalence of DM, including subjects who selfreported, was $32.0 \%$; the prevalence using WHO criteria was $27.4 \%$ (age-adjusted $20.1 \%)$, being higher in women $(p<0.001)$ and with advancing age ( $p$-trend $<0.001$ ). Prediabetes (IFG or IGT) was found in $29.1 \%$ of individuals (age-adjusted $17.6 \%$ ) and IFG in $14.4 \%$ (age-adjusted 8.0\%); the prevalence of impaired fasting glucose was also higher with advancing age ( $p$-trend $<0.001$ ), with a peak in the 55 - 64-year age group. Insulin resistance (HOMA-IR) was found in $55.5 \%$ of participants (age-adjusted $49.6 \%$ ); surprisingly, 36.6\% (ageadjusted $28.2 \%$ ) of the participants in the youngest age group (15- 24 years) were also so classified (Table 3 ).

Hypercholesterolaemia was found in $57.0 \%$ of subjects, hypertriglyceridaemia in $42.2 \%$ and raised LDL cholesterol in $23.7 \%$. A low concentration of HDL cholesterol was found in $43.3 \%$ of subjects, especially in women (48.1\%) as opposed to men (30.1\%). Of note is that even in the 15 - 24-year age group, $12.9 \%$ had elevated triglyceride levels and $35.1 \%$ had low HDL cholesterol levels. Diabetic dyslipidaemia (a combination of elevated triglycerides and LDL cholesterol, as well as low HDL cholesterol) was found in $4.4 \%$ of the sample, the prevalence being higher in men $(6.6 \%)$ than in women (3.6\%). Of the subjects $52.3 \%$ of males and $14.6 \%$ of females were current smokers, the figure for subjects in the 15 - 24-year age group being $26.7 \%$.

\section{Risk factor analysis}

Age, total serum cholesterol, serum triglyceride, HDL cholesterol, waist circumference, BMI, blood pressure, smoking and HOMA values were used in a bivariate analysis for DM and hypertension. The BMI, blood pressure, smoking and HOMA values were not significant on bivariate analysis, and were subsequently excluded from the logistic regression model. In multiple logistic regression analysis, age, total serum cholesterol, serum triglyceride, HDL cholesterol and waist circumference were entered into the predictive

\begin{tabular}{|c|c|c|c|c|c|}
\hline Variable & B & Wald & $p$-value & OR & 95\% CI \\
\hline \multicolumn{6}{|l|}{ Diabetes } \\
\hline Waist (cm) & 0.016 & 12.8 & $<0.001$ & 1.02 & $1.007-1.026$ \\
\hline $\operatorname{Tg}(\mathrm{mmol} / \mathrm{L})$ & 0.576 & 61.1 & $<0.001$ & 1.80 & $1.11-1.59$ \\
\hline Age & 0.02 & 5.125 & $<0.005$ & 1.06 & $1.044-1.070$ \\
\hline \multicolumn{6}{|l|}{ Hypertension } \\
\hline Waist (cm) & 0.037 & 60 & $<0.001$ & 1.04 & $1.03-1.047$ \\
\hline $\mathrm{FBG}(\mathrm{mmol} / \mathrm{L})$ & 0.017 & 20.8 & $<0.001$ & 1.02 & $1.069-1.081$ \\
\hline $\mathrm{Tg}(\mathrm{mmol} / \mathrm{L})$ & 0.162 & 8.028 & 0.05 & 1.18 & $1.01-1.4$ \\
\hline Age & 0.070 & 133 & $<0.001$ & 1.07 & $1.06-1.086$ \\
\hline \multicolumn{6}{|c|}{$\begin{array}{l}\mathrm{B}=\text { used to predict the dependent variable from the independent variable and presented } \\
\text { in log-odds units; Wald statistic = used to assess the significance of the coefficients and } \\
\text { contribution of individual predictors in a given model; Waist = waist circumference; } \\
\mathrm{Tg}=\text { triglyceride; } \mathrm{FBG}=\text { fasting blood glucose. }\end{array}$} \\
\hline
\end{tabular}

model. The significant independent risk factors associated with DM included age, waist circumference and serum triglyceride. For hypertension, the same risk factors were independent predictors with the addition of fasting plasma glucose (Table 4).

\section{Discussion}

This is the first study of SA Indians to document an extremely high $\mathrm{CV}$ risk factor prevalence, and it highlights the evolving epidemic of $\mathrm{CV}$ risk in teenagers and young adults in this population. Asian Indians across the diaspora are known to have high CV risk. ${ }^{[13]} \mathrm{A}$ previous diabetes epidemiology study reported over 20 years ago in the greater Durban area showed lower prevalences of hypertension (14\%) and DM (13\%). ${ }^{[2]}$ Our study shows that the magnitude of change over two decades has been of epidemic proportions in subjects with similar environmental exposure.

The prevalences of hypertension (47.5\%) and DM (agestandardised 20.1\%), in particular, are also strikingly high when compared with other population groups in SA. Stewart et al. ${ }^{[14]}$ reported a $33 \%$ prevalence of hypertension and a $5 \%$ prevalence of DM in their Soweto cohort (black African), with a $28 \%$ prevalence of DM in the coloured community. ${ }^{[15]}$

Furthermore, the high number of participants who were currently smoking, particularly in the youngest age group, is concerning since these individuals already had multiple risk factors in the presence of smoking, which is known to exert a detrimental multiplicative effect on CV risk. ${ }^{[16]}$

Our study shows a high prevalence of hypercholesterolaemia (57\%), raised triglyceride levels (42.2\%), raised LDL cholesterol levels (23.7\%) and low HDL cholesterol levels (43.3\%). The low HDL cholesterol and raised triglyceride levels are in keeping with the characteristic dyslipidaemia pattern seen in Asian Indians, which together with the 'thin-fat Asian phenotype' (low BMI and raised waist circumference) is thought to contribute to the development of excess CVD in this population group. ${ }^{[17]}$ Our findings are in direct contrast to this concept, since most of our subjects had generalised (both abdominal and total body) obesity. This, together with the emergence of multiple CV risk factors, including atherogenic dyslipidaemia, in the younger age group, predicts early onset of coronary heart disease ${ }^{[4]}$ and is likely to impose a greater burden of $\mathrm{CV}$ disease in the near future, as these individuals reach adulthood.

\section{Study strengths and limitations}

The study is limited by its cross-sectional design, which rendered us unable to observe or to report on trends associated with CV events that could have developed over time. Another limitation was the low response rates of male participants, which means that the data on men should be interpreted with caution; however, this has been reported in other SA population studies. ${ }^{[1,15]}$ In addition, limited participation was noted in the younger age groups, introducing a potential selection bias for non-working participants. The strength of this study is that it was a population-based study that also included a small sample of younger individuals and was able to show that $\mathrm{CV}$ risk was significantly high in this age group.

\section{Conclusion}

The extremely high prevalence of risk factors in our study explains the emergence of premature coronary heart disease in the community studied and calls for urgent and aggressive intervention measures aimed at diet, physical activity and cessation of smoking at schoolgoing level. A longitudinal follow-up study of these participants to ascertain the degree of $\mathrm{CV}$ risk associated with the marked changes in phenotype is urgently required. 
Acknowledgements. We thank our participants from the Phoenix community.

Sources of support. This research was made possible in part by a grant from the WHO (Africa Division) and financial support from Servier and Pfizer laboratories. We also wish to acknowledge General Motors (SA) for use of their bus to transport our participants.

Disclaimer. The views expressed in this article are the authors' own and not the official position of the Durban University of Technology or the University of KwaZulu-Natal or the funders.

\section{References}

1. International Diabetes Federation Atlas (2013). http://www.idf.org/sites/default/files/EN_6E_Ch2 the_Global_Burden.pdf./26/1/14 (accessed 26 January 2014).

2. Omar MAK, Seedat MA, Dyer RB, Motala AA, Knight LT, Becker PJ. South African Indians show a high prevalence of NIDDM and bimodality in plasma glucose distribution patterns. Diabetes Care 1994;17(1):70-73. [http://dx.doi.org/10.2337/diacare.17.1.70]

3. Motala AA, Omar MAK, Gouws E. High risk of progression to NIDDM in South-African Indians with impaired glucose tolerance. Diabetes 1993;42(4):556-563. [http://dx.doi.org/10.2337/ diab.42.4.556]

4. Ranjith N, Pegoraro RJ, Naidoo DP, Shanmugam R, Rom L. Genetic variants associated with insulin resistance and metabolic syndrome in young Asian Indians with myocardial infarction. Metab Synd Relat Disord 2008;6(3):209-214. [http://dx.doi.org/10.1089/met.2008.0023]

5. Seedat YK, Mayet FG, Khan S, Somers SR, Joubert G. Risk factors for coronary heart disease in the Indians of Durban. S Afr Med J 1990;78(8):447-454.

6. Kish L. Survey Sampling. New York: Wiley-Interscience, 1995. http://apps.who.int/iris/bitstream/10665/68346/1/ WHO_NMH_CCS_03.03.pdf?ua=1/ (accessed 22 February 2008)
8. Barba C, Cutter J, Deurenberg P, et al. Appropriate body-mass index for Asian populations and its implications for policy and intervention strategies. Lancet 2004;363(9403):157-163. [http://dx.doi. implications for policy and interventions
$\mathrm{org} / 10.1016 / \mathrm{s} 0140-6736(03) 15268-3]$

9. Chobanian AV, Bakris GL, Black HR, et al. Seventh Report of the Joint National Committee Chobanian AV, Bakris GL, Black HR, et al. Seventh Report of the Joint National Committee
on Prevention, Detection, Evaluation, and Treatment of High Blood Pressure. Hypertension on Prevention, Detection, Evaluation, and Treatment of High Blood Press
2003;42(6):1206-1252. [http://dx.doi.org/10.1161/01.hyp.0000107251.49515.c2]

10. Expert Panel on Detection, Evaluation, and Treatment of High Blood Cholesterol in Adults. Executive Summary of the Third Report of the National Cholesterol Education Program (NCEP) Expert Panel on Detection, Evaluation, and Treatment of High Blood Cholesterol in Adults (Adult Treatment Panel III). JAMA 2001;285(19):2486-2497. [http://dx.doi.org/10.1001/jama.285.19.2486]

11. World Health Organization and International Diabetes Federation. Definition and diagnosis of diabetes mellitus and intermediate hyperglycemia: Report of a WHO/IDF consultation. 2005. http:// apps.who.int/iris/bitstream/10665/43588/1/9241594934_eng.pdf (accessed 16 July 2012).

12. Ascaso JF, Pardo S, Real JT, Lorente RI, Priego A, Carmena R. Diagnosing insulin resistance by simple quantitative methods in subjects with normal glucose metabolism. Diabetes Care 2003;26(12):3320-3325. [http://dx.doi.org/10.2337/diacare.26.12.3320]

13. Yusuf S, Reddy S, Ounpuu S, Anand S. Global burden of cardiovascular diseases: Part II: Variations in cardiovascular disease by specific ethnic groups and geographic regions and prevention strategies. Circulation 2001;104(23):2855-2864. [http://dx.doi.org/10.1161/hc4701.099488]

14. Stewart S, Carrington M, Pretorius S, Methusi P, Sliwa K. Standing at the crossroads between new and historically prevalent heart disease: Effects of migration and socio-economic factors in the Heart of Soweto cohort study. Eur Heart J 2010;32(4):492-499. [http://dx.doi.org/10.1093/eurheartj/ ehq439]

15. Erasmus RT, Soita DJ, Hassan MS, et al. High prevalence of diabetes mellitus and metabolic syndrome in a South African coloured population: Baseline data of a study in Bellville, Cape Town. S Afr Med J 2012;102(11):841-844. [http://dx.doi.org/10.7196/SAMJ.5670]

6. Mikhailidis DP, Papadakis JA, Ganotakis ES. Smoking, diabetes and hyperlipidaemia. J R Soc Promot Health 1998;118(2):91-93. [http://dx.doi.org/10.1177/146642409811800209]

17. Enas EA, Chacko V, Pazhoor SG, Chennikkara H, Devarapalli HP. Dyslipidemia in South Asian patients. Curr Atheroscler Rep 2007:9(5):367-374. [http://dx.doi.org/10.1007/s11883-007-0047-y]

Accepted 16 November 2015. 